\title{
ÉDITORIAL
}

\section{Y a-t-il une phytothérapie immunostimulante ?}

\author{
P. Goetz \\ (C) Lavoisier SAS 2018
}

En préparant le thème de mon intervention ${ }^{1}$ sur les effets antitumoraux des plantes immunomodulatrices et entendant un enseignant douter de l'effet immunostimulant, $j$ 'en suis venu à me demander si la phytothérapie immunostimulante existe ou non.

Comme je l'ai toujours pensé et dit, les immunostimulants ne peuvent agir qu'en stimulant les médiateurs immunitaires.

Les démonstrations portant particulièrement sur Echinacea montrent bien qu'il n'y a pas une action directe sur les microbes, mais sur des éléments circulants comme les lymphocytes ou les macrophages. Nous savons aujourd'hui que l'effet de l'échinacée est réduit dans le temps et qu'il faut renouveler la dose chaque jour. Les états d'hypoleucocytose sont une contre-indication. L'effet d'échinacée est rapide, mais il faut une imprégnation pour obtenir tous les effets. Une mobilisation des polynucléaires, des lymphocytes, des euglobulines, des macro- et microphages, anti-TNF-alpha, etc. ne se fait pas d'emblée.

Mes propres constatations cliniques m'indiquent qu'une immunostimulation pour la pathologie hivernale doit être débutée en période préhivernale et maintenue jusqu'à une période au climat plus clément.

Les expérimentations allemandes, menées scientifiquement, ont bien mesuré ce mode d'action et mode de traitement. Rappelons que l'une des premières études concerne la réduction des récidives de vaginite à Trichomonas par la prise d'Echinacin ${ }^{\circledR}$ (Echinacea purpurea plante totale dans de l'alcool à titre de conservateur).

L'exercice de la phytothérapie se fait bien en cherchant et croisant des informations qui ne se limitent pas à notre exigu Hexagone.

C'est ce que propose de relayer votre revue de phytothérapie pour parfaire vos connaissances.

P. Goetz $(\bowtie)$

DU de phytothérapie, Paris-XIII, F-93017 Bobigny cedex, France

e-mail : paul.goetz@wanadoo.fr

\footnotetext{
${ }^{1}$ Présentée le samedi 7 avril, lors du colloque médical, Les rencontres de phytothérapie et médecines complémentaires. «Soins de support et accompagnement des cancers ", organisé à Courbevoie par SavoirSport-Santé et présidé par B. Chemouny, directeur de l'enseignement du DU Phyto et Aroma de l'Université Paris-XIII.
} 